\title{
Descripción de larvas mysis de tres especies de camarones mesopelágicos del género Gennadas (Decapoda: Aristeidae) en aguas del Pacífico sudoriental
}

\author{
Jorge Rivera y Guillermo Guzmán \\ Departamento de Ciencias del Mar, Universidad Arturo Prat, Casilla 121, Iquique, Chile \\ E-mail: riveramon@mixmail.com
}

Recibido: 20 diciembre 2001; versión corregida: 11 septiembre 2002; aceptado: 7 octubre 2002

\begin{abstract}
RESUMEN. A nivel mundial solo se han reportado los estadios larvales de dos especies de camarones del género Gennadas, ambas del océano Atlántico. Durante el transcurso de un crucero de investigación realizado desde Caldera, costa norte de Chile, hasta Isla de Pascua, se colectaron tres morfotipos de estadios mysis, pertenecientes a este género, ninguno de ellos pertenece a alguna de las especies previamente descritas. Se entregan las descripciones de esos especímenes y se comparan con las especies previamente descritas.
\end{abstract}

Palabras claves: Gennadas, estadio de mysis, descripción, Pacífico sudeste.

\section{Description of mysis larvae of three mesopelagic shrimp species of the genus Gennadas (Decapoda: Aristeidae) from the southeastern Pacific waters}

\begin{abstract}
Only two description of larval stage of shripms belonging to Gennadas genus are reported to this time, both of these to Atlantic oceans. During a survey off coast from Caldera, northern Chile, to Easter Island, three morphotypes of mysis stage of shrimp's belonging to Gennadas genus were collected, none of these showed some resemblance with the descriptions in the literature. The results of the descriptions of these samples and his comparation with previously knowed species are showed here.
\end{abstract}

Key words: Gennadas, mysis stages, description, southeastern Pacific.

\section{INTRODUCCIÓN}

Los camarones mesopelágicos del género Gennadas (Bate, 1881) tienen amplia distribución en ambos hemisferios y cumplen un rol importante en la trofodinámica de los ecosistemas marinos oceánicos, junto a otros camarones Peneidos y Carideos (Foxton, 1970; Omori, 1974). Trabajos realizados en el hemisferio norte entregan información bioecológica sobre el género Gennadas, registrando la batimetría y migraciones verticales de juveniles y adultos en el micronecton, considerándolos como fuertes migradores verticales (Heffermman y Hopkins, 1981; Andersen y Sardou, 1992).

Este grupo de organismos está bien representado en las aguas del hemisferio norte (Hanamura, 1981; Kikuchi y Nemoto, 1986, 1991; Hendrickx y Estrada-Navarrete, 1989, 1996; Wicksten y Hendrickx, 1992). Sin embargo son escasos los an- tecedentes acerca de su distribución en aguas del Pacífico sudeste. En aguas chilenas se ha registrado la presencia de ocho especies pertenecientes a la subfamilia Benthesicyminae, cinco de las cuales pertenecen al género Gennadas (Guzmán y Wicksten, 2000).

La falta de trabajos en este grupo de decápodos, es más notoria aún en su desarrollo larval debido a la gran dificultad de mantener especimenes vivos en laboratorios (K. Konishi, com. pers, y K. Hashizume, com. pers.). Los trabajos de Gurney (1924) y Heldt (1938) describieron los estadios protozoea y mysis de Gennadas elegans Smith desde muestras del plancton. Posteriormente, Subrahmanyam y Gunter (1970) reportaron un estadio protozoea de una especie indeterminada del género Gennadas colectada en la costa de Mississipi 
en el Atlántico norte. Criales y McGowan (1993) realizaron una descripción de los cuatro estadios mysis para una especie del género Gennadas recolectada del plancton y que probablemente correspondan a G. valens Smith distribuida en el Atlántico noroeste.

En Chile no se han realizado descripciones parciales ni completas de especies de la superfamilia Penaeoidea (Wehrtmann y Báez, 1997), y por lo tanto, el objetivo principal de este trabajo es dar a conocer la descripción parcial de las larvas de tres morfotipos en estadios mysis del género Gennadas, capturadas entre Caldera e Isla de Pascua.

\section{MATERIALES Y MÉTODOS}

Las larvas se colectaron en el material mesopelágico capturado durante el desarrollo del Proyecto CIMAR-5 Islas Oceánicas, en un transecto entre Caldera $\left(27^{\circ} 00^{\prime} \mathrm{S} ; 70^{\circ} 52^{\prime} \mathrm{W}\right)$ e Isla de Pascua $\left(27^{\circ} 20^{\prime} \mathrm{S} ; 109^{\circ} 40^{\prime} \mathrm{W}\right)$ efectuado en octubre y noviembre de 1999, a bordo del buque oceanográfico "Vidal Gormaz", mediante una red de arrastre horizontal y vertical Isaacs Kids Midwater Trawl (IKMT) de $9 \mathrm{~m}^{2}$ de abertura de boca, túnel de $10 \mathrm{~m}$ de largo con malla de $10 \mathrm{~mm}$ y un copo de 1000 micras.

El transecto analizado comprendió 13 estaciones oceánicas, con una profundidad aproximada de $500 \mathrm{~m}$ por estación. Estas estaciones se localizaron a 50, 150, 280, 440, 600, 760, 920, 1080, 1240, 1400, 1560,1720 y $2000 \mathrm{mn}$ de la costa desde Caldera hasta Isla de Pascua, incluyendo lances diurnos y nocturnos (Tabla 1).

Las larvas se identificaron como pertenecientes al género Gennadas, de acuerdo a los trabajos descriptivos de Heldt (1938), y Criales y McGowan (1993), donde se caracterizan los patrones morfológicos generales, tales como el caparazón con un largo rostro, con o sin dentículos sobre él, con una espina en su base, espinas supraorbital y branquiostegal largas. El abdomen con espinas dorsales siendo siempre la del segundo segmento más larga. Telson profundamente hendido formando una furca terminal. Primer par de pleópodos globulares con pequeños procesos espiniformes, muy peculiares. Maxílula con endópodo trisegmentado durante la mysis I, bisegmentado en la mysis II y sin segmentos en las mysis III y IV. Maxila con ambos enditos, basal y coxal bilobulados. Maxilípedo 1 con epipodito prolongado en forma aplanada y protopodito bilobulado, con su endito basal más prolongado que el endito coxal (Figs. 1a a 1g).

Los especímenes se preservaron en una solución de formalina al 5\% neutralizada con bórax y se tiñeron con azul de metileno para facilitar su estudio, caracterizando tres morfotipos distintos (Gennadas sp. 1, sp. 2, sp. 3) (sensu Díaz, 1998). El número total de larvas de cada morfotipo se registró en cada estación (Tabla 2).

Tabla 1. Datos de las estaciones del crucero CIMAR 5 con ocurrencia de larvas del género Gennadas (octubrenoviembre de 1999).

Table 1. Stations data of CIMAR 5 cruise, with occurrence of the genus Gennadas larvae (October-November 1999).

\begin{tabular}{|lcccc|}
\hline Estación & Fecha & Latitud $(\mathbf{S})$ & Longitud $(\mathbf{W})$ & Profundidad (m) \\
\hline E-5 & $16-10-99$ & $26^{\circ} 59^{\prime} 41$ & $71^{\circ} 46^{\prime} 00$ & 500 \\
E-7 & $17-10-99$ & $27^{\circ} 00^{\prime} 52$ & $73^{\circ} 37^{\prime} 26$ & 500 \\
E-9 & $18-10-99$ & $27^{\circ} 03^{\prime} 37$ & $76^{\circ} 05^{\prime} 28$ & 500 \\
E-11 & $19-10-99$ & $27^{\circ} 00^{\prime} 07$ & $79^{\circ} 05^{\prime} 05$ & 500 \\
E-13 & $20-10-99$ & $27^{\circ} 00^{\prime} 08$ & $82^{\circ} 05^{\prime} 05$ & 500 \\
E-15 & $21-10-99$ & $27^{\circ} 00^{\prime} 14$ & $85^{\circ} 03^{\prime} 41$ & 500 \\
E-16 & $21-10-99$ & $27^{\circ} 00^{\prime} 21$ & $86^{\circ} 33^{\prime} 14$ & 500 \\
E-18 & $22-10-99$ & $27^{\circ} 00^{\prime} 24$ & $89^{\circ} 34^{\prime} 02$ & 500 \\
E-21 & $21-10-99$ & $27^{\circ} 00^{\prime} 08$ & $94^{\circ} 05^{\prime} 49$ & 500 \\
E-23 & $25-10-99$ & $26^{\circ} 59^{\prime} 55$ & $97^{\circ} 05^{\prime} 55$ & 500 \\
E-25 & $25-10-99$ & $27^{\circ} 04^{\prime} 02$ & $100^{\circ} 03^{\prime} 56$ & 500 \\
E-27 & $26-10-99$ & $27^{\circ} 00^{\prime} 37$ & $103^{\circ} 05^{\prime} 45$ & 500 \\
E-30 & $28-10-99$ & $27^{\circ} 00^{\prime} 37$ & $107^{\circ} 35^{\prime} 00$ & 500 \\
\hline
\end{tabular}


Las larvas de cada estadio mysis se midieron con un ocular graduado, considerando la longitud total (LT), desde el margen orbital anterior hasta la escotadura del telson; la longitud del caparazón (LC) medida desde el borde anterior del caparazón hasta el margen medio posterior del mismo, y la longitud del rostro (LR) desde la punta del rostro hasta el margen medio anterior del caparazón (Tabla 3). La disección, descripción y esquematización de los especímenes, se realizó mediante un microscopio con cámara clara.
Los especímenes en estadio de mysis de los tres morfotipos de Gennadas, se depositaron en el Museo del Mar del Departamento de Ciencias del Mar, de la Universidad Arturo Prat de Iquique, Chile, bajo los códigos MUAP(CD) indicados en los resultados.

\section{RESULTADOS}

Se analizó un total de 42 larvas pertenecientes a tres especies no identificadas, asignadas al género

Tabla 2. Larvas de Gennadas spp. en las estaciones del crucero CIMAR 5 (octubre-noviembre 1999).

Table 2. Larvae of Gennadas spp. in the stations of CIMAR 5 cruise (October-November 1999).

\begin{tabular}{|lcccccccccccccc|}
\hline Morfotipo E-5 & E-7 & E-9 & E-11 & E-13 & E-15 & E-16 & E-18 & E-21 & E-23 & E-25 & E-27 & E-30 & Total \\
\hline sp. 1 & 1 & 6 & 6 & & 1 & & 2 & 1 & & & & & & 17 \\
sp. 2 & 1 & & & & & 4 & 3 & & 1 & 4 & 3 & & 16 \\
sp. 3 & & & & & & & & 2 & & 1 & 1 & 2 & 3 & 9 \\
\hline
\end{tabular}

Tabla 3. Mediciones de los morfotipos de larvas del género Gennadas. Longitud total (LT), Longitud del caparazón (LC) y Longitud del rostro (LR) en mm. Número total de larvas por cada estadio (N) y número de larvas disectadas (n).

Table 3. Measurements of larvae morphotypes belong to genus Gennadas. Total length (LT), Caparace length (LC), Rostrum length (LR) in mm. Number of larvae reported by each stage (N) and number of larvae dissected (n).

\begin{tabular}{|c|c|c|c|c|}
\hline Morfotipo & $\begin{array}{c}\text { Mysis I } \\
(\mathbf{m m})\end{array}$ & $\begin{array}{c}\text { Mysis II } \\
(\mathbf{m m})\end{array}$ & $\begin{array}{c}\text { Mysis III } \\
\quad(\mathrm{mm})\end{array}$ & $\begin{array}{r}\text { Mysis IV } \\
\quad(\mathbf{m m})\end{array}$ \\
\hline \multicolumn{5}{|l|}{ sp. 1} \\
\hline LT & $3,24-3,36$ & $3,60-3,94$ & $4,30-4,41$ & $4,98-5,05$ \\
\hline LC & $0,80-0,85$ & $0,96-1,05$ & $1,10-1,18$ & $1,21-1,38$ \\
\hline LR & $1,12-1,28$ & $1,30-1,61$ & $1,64-2,04$ & $2,00-2,25$ \\
\hline $\mathbf{N}$ & 7 & 4 & 3 & 3 \\
\hline $\mathbf{n}$ & 4 & 2 & 2 & 2 \\
\hline \multicolumn{5}{|l|}{ sp. 2} \\
\hline LT & $3,30-3,50$ & $4,07-4,58$ & $5,50-5,81$ & $6,00-6,69$ \\
\hline LC & $0,90-1,07$ & $1,12-1,37$ & $1,93-2,02$ & $2,26-2,56$ \\
\hline LR & $0,90-1,00$ & $1,14-1,30$ & $1,37-1,53$ & $2,05-2,10$ \\
\hline $\mathbf{N}$ & 9 & 3 & 2 & 2 \\
\hline n & 5 & 2 & 1 & 1 \\
\hline \multicolumn{5}{|l|}{ sp. 3} \\
\hline LT & - & $3,50-3,84$ & $4,90-5,36$ & $6,00-6,05$ \\
\hline LC & - & $0,80-0,90$ & $1,12-1,28$ & 1,42 \\
\hline LR & - & $1,10-1,14$ & $1,67-1,90$ & $1,60-1,63$ \\
\hline $\mathbf{N}$ & 0 & 3 & 4 & 2 \\
\hline $\mathbf{n}$ & 0 & 1 & 2 & 1 \\
\hline
\end{tabular}


Gennadas por los caracteres descritos en la metodología. Estas larvas correspondieron a los estadios mysis I a IV del ciclo de vida descrito para este grupo. Las larvas más abundantes pertenecieron a Gennadas sp. 1 (17 larvas), seguida de Gennadas sp. 2 (16 larvas) y la menos abundante fue Gennadas sp. 3 (9 larvas).

\section{Gennadas sp. 1 (Figs. 2a a 2f)}

Material examinado: cuatro mysis que variaron entre 3,24 y 5,15 mm de longitud total (LT); el largo del caparazón (LC) se incrementó en cada estadio desde 0,85 en mysis I a 1,60 en mysis IV. Código: MUAP(CD)-0371.

Diagnosis: Caparazón liso, con un surco cervical y submarginal marcado; rostro largo armado con pequeños dentículos en la porción proximal (siendo estos más notorios en los últimos estadios), con una larga espina en su base; espinas supraorbital y branquiostegal largas (Fig. 2a). Segundo pleonito con una espina dorsal extremadamente larga incrementando su largo en cada estadio; segmentos tres y seis con espinas sobre el borde posterodorsal de mediano tamaño; segmentos cuatro y cinco con espinas dorsales pequeñas; del primer al sexto segmento con espinas laterales de las cuales la del primer somito es la más larga; sexto segmento con un par de espinas ventrolaterales. Las espinas laterales del tercero al sexto pleonito y las ventrolaterales del sexto segmento se reducen en los estadios mysis III y IV (Fig. 2a). Telson largo y estrecho, profundamente hendido, formando una furca con seis pares de espinas, de las cuales el par medio son las más largas; con un par de espinas laterales largas ubicadas en el medio del telson durante la mysis I, reduciéndose y desapareciendo en los estadios posteriores; urópodos largos y desarrollados completamente desde la mysis I, exópodos con espina externa (Fig. 2b).

\section{Gennadas sp. 2 (Figs. 3a a 3f)}

Material examinado: cuatro mysis, largo total (LT) entre 3,35 y 6,69 mm; el largo del caparazón (LC) se incrementó en cada estadio desde 1,05 en mysis I a 2,56 mm en mysis IV. Código: MUAP(CD)-0372.

Diagnosis: Caparazón con los surcos cervical, hepático, branquiocardiaco y submarginal pronunciados; rostro largo armado dorsalmente con dentículos a lo largo de éste (siendo más evidentes en los últimos estadios); espinas supraorbital y branquiostegal largas (Fig. 3a). Abdomen con el primer y segundo pleonito con espinas dorsales lar- gas, siendo la del segundo segmento siempre más larga; tercero al sexto segmento con espinas dorsales de similar tamaño sobre una carena dorsal poco definida; los seis segmentos con espinas laterales durante las mysis I y II, desapareciendo sobre los somito tercero y cuarto en las subsiguientes mysis; el sexto somito con un par de espinas ventrolaterales durante la mysis I, las cuales se reducen en los posteriores estadios, junto con la espina lateral de este (Fig. 3a). Telson largo y estrecho, profundamente hendido formando dos ramas con ocho pares de espinas terminales durante las mysis I y II, reduciéndose a siete pares en los estadios III y IV, las dos ramas terminan con las espinas más largas del telson; poseen un par de largas espinas laterales ubicadas en el medio, reduciéndose en los estadios mysis III y IV. Urópodos largos completamente desarrollados, exópodos con setas externas desde la mysis I (Fig. 3b).

\section{Gennadas sp. 3 (Figs. 4 a a 4 f)}

Material examinado: tres mysis cuyo largo total (LT) fluctuó entre 3,80 y 6,05 mm; el largo del caparazón (LC) aumentó en cada estadio de 0,90 en mysis II a 1,42 mm en mysis IV. Código: MUAP(CD)0373.

Diagnosis: Caparazón liso, con un profundo surco cervical y un leve surco submarginal; rostro largo armado con dentículos y con una espina basal larga; espina supraorbital larga y espina branchiostegal ligeramente larga (Fig. 4a). Abdomen con el segundo segmento con espina dorsal larga y con pequeñas espinas sobre el borde posterodorsal del primero, tercero, cuarto y quinto segmentos; sexto segmento con una espina dorsal dos veces el tamaño de las espinas anteriores, reduciéndose en las mysis III y IV; espinas laterales en el primer y segundo segmento; el sexto pleonito con un par de espinas ventrolaterales notorias durante las mysis I y II, las cuales se reducen en los estadios posteriores (Fig. 4a). Telson largo y profundamente hendido, formando dos ramas que terminan en una larga espina; con dos pares de pequeñas espinas laterales en el extremo distal del telson y con un par de espinas laterales de mayor tamaño ubicadas en el medio de éste, las que se reducen en las mysis III y IV (Fig. 4b). 

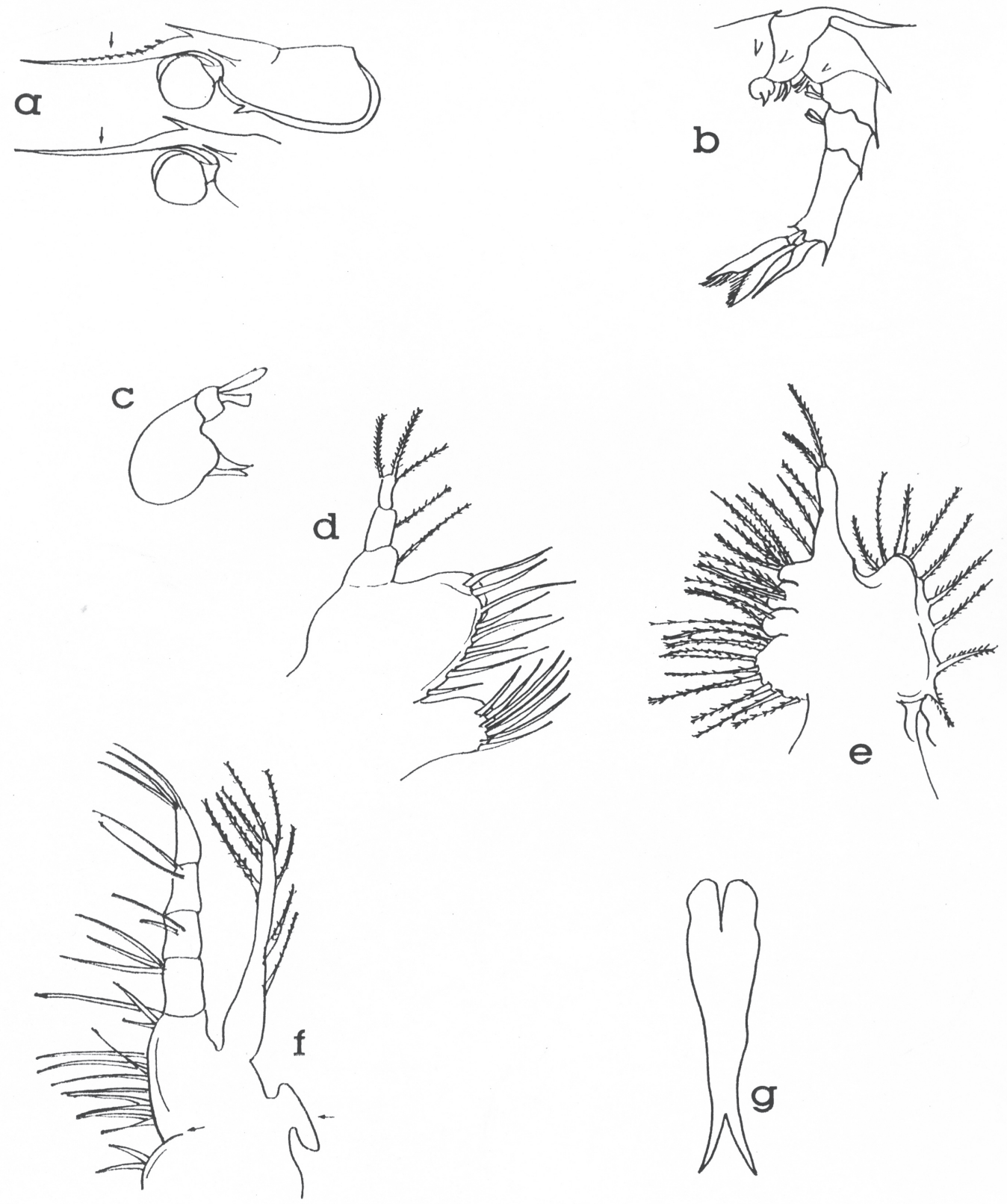

Figura 1. Patrones morfológicos de las larvas mysis de Gennadas spp. a) caparazón, b) abdomen, c) primer pleópodo, d) maxílula de mysis I, e) maxila, f) maxilípedo 1, g) telson.

Figure 1. Morfological patterns in the mysis larvae of Gennadas spp. a) caparace, b) abdomen, c) first pleopod, d) maxillule of mysis I, e) maxilla, f) maxilliped 1, g) telson. 


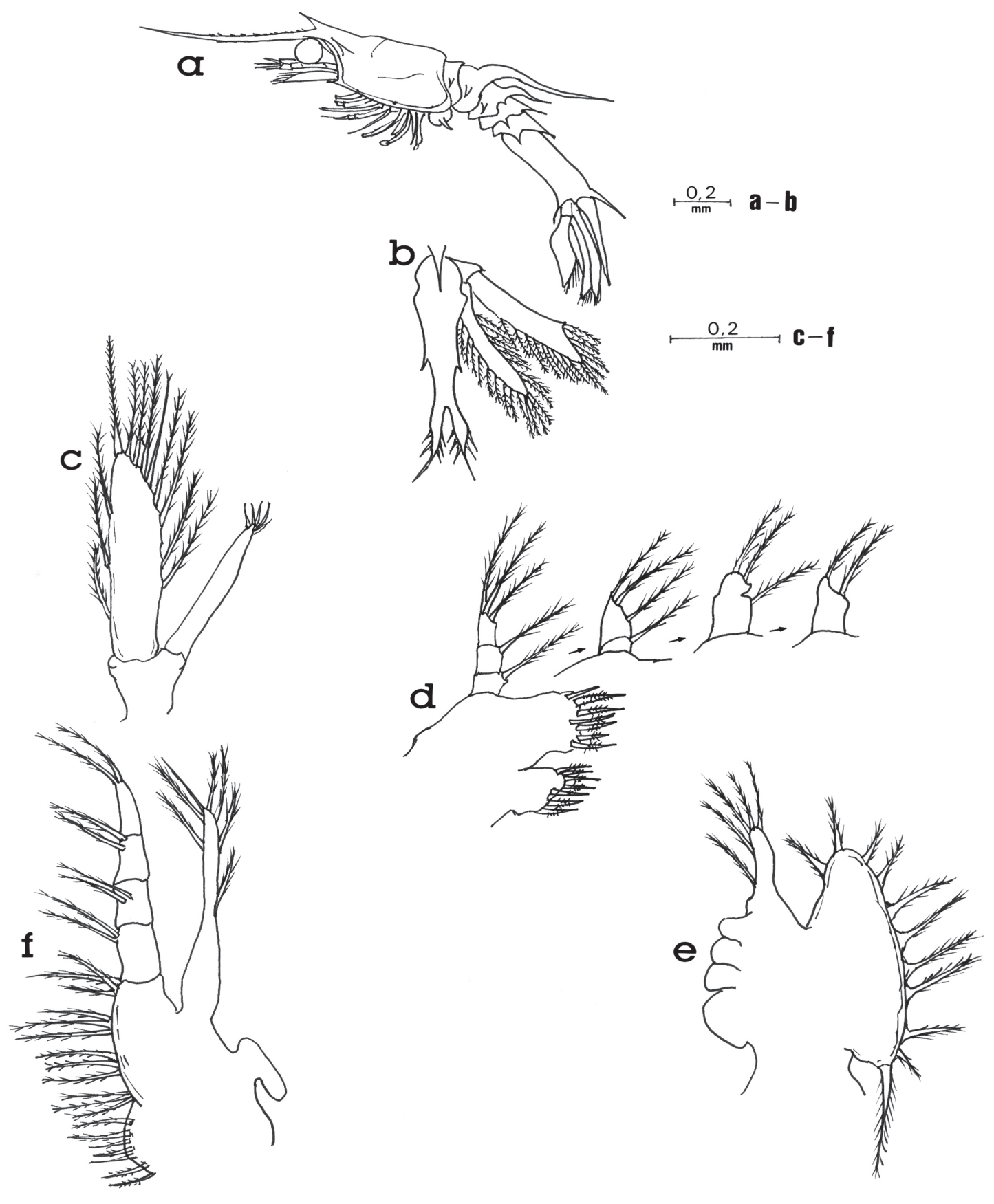

Figura 2. Mysis I de Gennadas sp. 1: a) vista lateral, b) telson, c) antena, d) maxílula (la flecha indica el desarrollo del endópodo de la maxílula desde mysis I a IV), e) maxila, f) maxilípedo 1. Escalas: 0,2 mm.

Figure 2. Mysis I of Gennadas sp. 1: a) lateral view, b) telson, c) antenna, d) maxillule (the arrow show the development of the endopod of the maxillule since mysis I to IV), e) maxilla, f) maxilliped 1. Bar scales: $0.2 \mathrm{~mm}$. 




Figura 3. Mysis I de Gennadas sp. 2: a) vista lateral, b) telson, c) antena, d) maxílula (la flecha indica el desarrollo del endópodo de la maxílula desde mysis I a IV), e) maxila, f) maxilípedo 1. Escalas: 0,2 mm.

Figure 3. Mysis I of Gennadas sp. 2: a) lateral view, b) telson, c) antenna, d) maxillule (the arrow show the development of the endopod of the maxillule since mysis I to IV), e) maxilla, f) maxilliped 1. Bar scales: $0.2 \mathrm{~mm}$. 


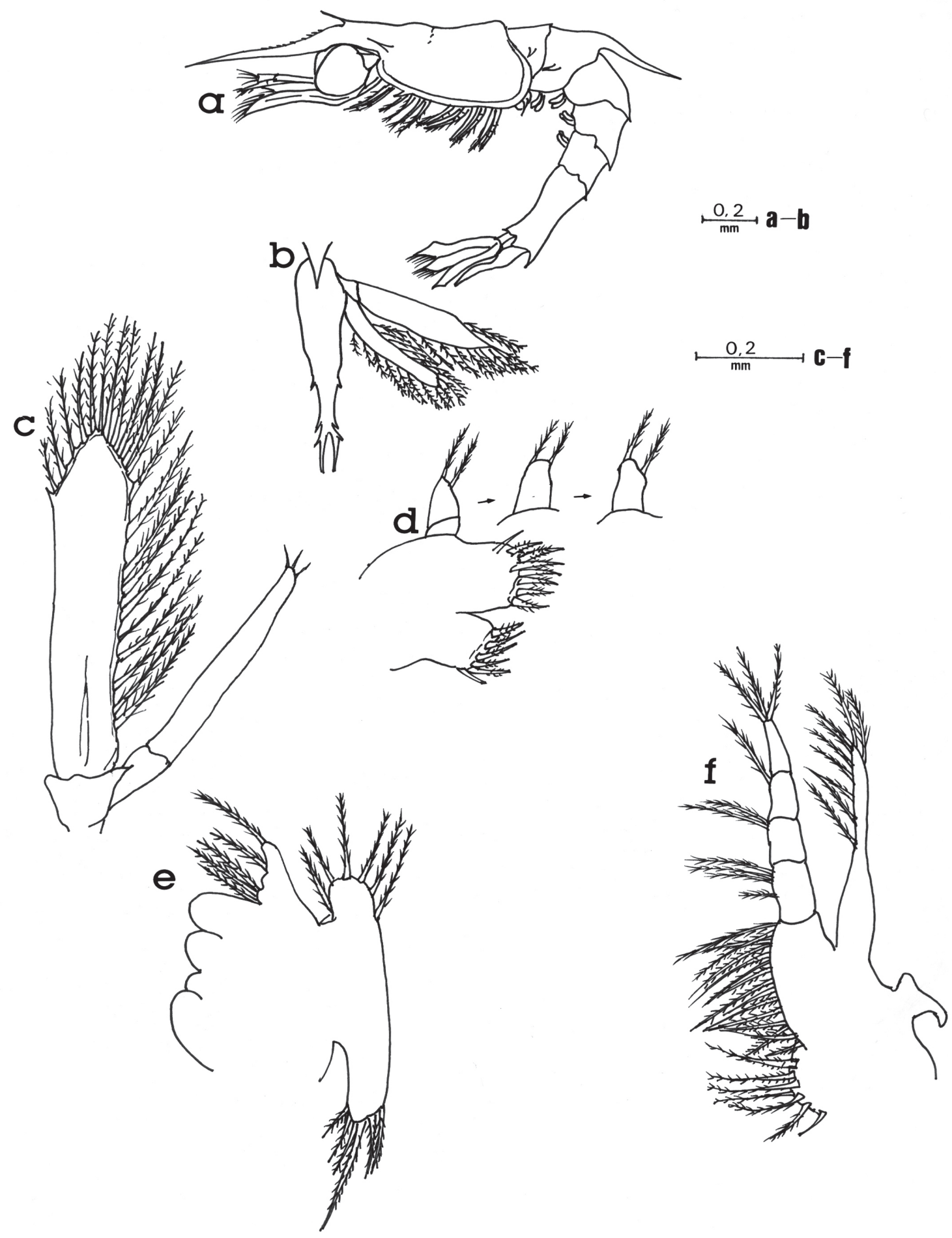

Figura 4. Mysis III de Gennadas sp. 3: a) vista lateral, b) telson, c) antena, d) maxílula (la flecha indica el desarrollo del endópodo de la maxílula desde mysis I a IV), e) maxila, f) maxilípedo 1. Escalas: 0,2 mm.

Figure 4. Mysis III of Gennadas sp. 3: a) lateral view, b) telson, c) antenna, d) maxillule (the arrow show the development of the endopod of the maxillule since mysis I to IV), e) maxilla, f) maxilliped 1. Bar scales: $0.2 \mathrm{~mm}$. 


\section{DISCUSIÓN}

Las larvas observadas en este trabajo, siguen los patrones morfológicos generales descritos por Gurney (1924) y Heldt (1938) para G. elegans Smith. Estos autores no presentan en sus trabajos una descripción completa de cada apéndice en los diferentes estadios, por lo cual no es posible realizar una comparación detallada con respecto a esta especie. En cambio Criales y McGowan (1993), trabajando con larvas que posiblemente correspondían a G. valens Smith, en la costa sur de Florida, realizaron una descripción que incluyó las características de cada apéndice para cada uno de los cuatro estadios de mysis.
Sin embargo, es posible establecer notables diferencias entre las larvas antes mencionadas con los tres morfotipos descritos en el presente trabajo. Estas diferencias se relacionaron principalmente con la espinación externa en el telson y en el abdomen (Tabla 4). Las mysis de Gennadas sp. 1, sp. 2 y sp. 3, poseen pequeñas espinas sobre el rostro, lo que las diferencia de las larvas de G. elegans y G. valens? registradas en el Atlántico norte, que carecen de este carácter durante el desarrollo de los estadios de mysis.

Esto indicaría una mayor variabilidad en la morfología externa en la fase larval que en la fase adulta, en las especies de este género. Debido a que en la fase adulta es muy difícil diferenciar una especie

Tabla 4. Comparación morfológica de los estadios mysis entre los diferentes morfotipos de Gennadas, sobre la base de su espinación externa. En el abdomen las espinas se indican por somito. ( ) espinas reducidas desapareciendo; * Fórmula de las espinas furcales: externas + terminales + internas

Table 4. Comparison of mysis stages in different morphotypes of Gennadas larvae on the basis of external spinations. The abdominal spines are showed by each somite. ( ) spines small or lacking; * External furcal spines formula: external + terminal + inner.

\begin{tabular}{|c|c|c|c|c|c|c|}
\hline & \multirow{2}{*}{\begin{tabular}{|c|} 
Dentículos \\
del \\
rostro \\
\end{tabular}} & \multicolumn{3}{|c|}{ Espinas del abdomen } & \multicolumn{2}{|c|}{ Espinas del telson } \\
\hline & & dorsal & lateral & ventrolateral & lateral & furcal* \\
\hline $\begin{array}{l}\text { Gennadas sp. } 1 \\
\text { Mysis I } \\
\text { Mysis II } \\
\text { Mysis III } \\
\text { Mysis IV } \\
\end{array}$ & $\begin{array}{l}\text { Presentes } \\
\text { Presentes } \\
\text { Presentes } \\
\text { Presentes }\end{array}$ & $\begin{array}{l}2 \text { al } 6 \\
2 \text { al } 6 \\
2 \text { al } 6 \\
2 \text { al } 6 \\
\end{array}$ & $\begin{array}{l}1 \text { al } 6 \\
1 \text { al } 6 \\
1 \text { al } 4 \\
1 \text { al } 3 \\
\end{array}$ & $\begin{array}{l}6 \\
6 \\
- \\
- \\
\end{array}$ & $\begin{array}{l}1 \\
1 \\
- \\
-\end{array}$ & $\begin{array}{c}2+1+3 \\
2+1+3 \\
(2)+1+(3) \\
(1)+1+(1)\end{array}$ \\
\hline $\begin{array}{l}\text { Gennadas sp. } 2 \\
\text { Mysis I } \\
\text { Mysis II } \\
\text { Mysis III } \\
\text { Mysis IV }\end{array}$ & $\begin{array}{l}\text { Presentes } \\
\text { Presentes } \\
\text { Presentes } \\
\text { Presentes }\end{array}$ & $\begin{array}{l}1 \text { al } 6 \\
1 \text { al } 6 \\
1 \text { al } 6 \\
1 \text { al } 6\end{array}$ & \begin{tabular}{|c|}
1 al 6 \\
1 al 6 \\
$1,2,5,6$ \\
$1,2,5,6$
\end{tabular} & $\begin{array}{c}6 \\
6 \\
(6) \\
-\end{array}$ & $\begin{array}{c}1 \\
1 \\
(1) \\
-\end{array}$ & $\begin{array}{l}1+1+6 \\
1+1+6 \\
0+1+6 \\
0+1+6\end{array}$ \\
\hline $\begin{array}{l}\text { Gennadas sp. } 3 \\
\text { Mysis I } \\
\text { Mysis II } \\
\text { Mysis III } \\
\text { Mysis IV }\end{array}$ & $\begin{array}{c}\text { - } \\
\text { Presentes } \\
\text { Presentes } \\
\text { Presentes }\end{array}$ & $\begin{array}{c}- \\
1 \text { al } 6 \\
\text { (1) al } 6 \\
2 \text { al } 6 \\
\end{array}$ & 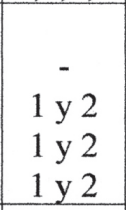 & $\begin{array}{c}- \\
6 \\
(6) \\
-\end{array}$ & $\begin{array}{c}- \\
3 \\
(2) \\
(2)\end{array}$ & $\begin{array}{c}- \\
0+1+0 \\
0+1+0 \\
0+1+0\end{array}$ \\
\hline $\begin{array}{l}\text { Gennadas elegans } \\
\text { Gurney (1924) } \\
\text { Mysis I } \\
\text { Mysis II } \\
\text { Mysis III } \\
\text { Mysis IV }\end{array}$ & $\begin{array}{l}\text { Ausentes } \\
\text { Ausentes } \\
\text { Ausentes } \\
\text { Ausentes }\end{array}$ & $\begin{array}{l}2 \text { al } 6 \\
2 \text { al } 6 \\
2 \text { al } 6 \\
2 \text { al } 6\end{array}$ & \begin{tabular}{|c|}
1 al 6 \\
1 al 6 \\
1 al (6) \\
1 al 5 \\
\end{tabular} & $\begin{array}{c}6 \\
6 \\
(6) \\
(6)\end{array}$ & $\begin{array}{l}1 \\
1 \\
1 \\
1\end{array}$ & $\begin{array}{l}1+1+2 \\
1+1+2 \\
1+1+2 \\
1+1+2\end{array}$ \\
\hline $\begin{array}{l}\text { Gennadas valens? } \\
\text { Criales y McGowan (1993) } \\
\text { Mysis I } \\
\text { Mysis II } \\
\text { Mysis III } \\
\text { Mysis IV }\end{array}$ & $\begin{array}{l}\text { Ausentes } \\
\text { Ausentes } \\
\text { Ausentes } \\
\text { Ausentes }\end{array}$ & $\begin{array}{l}2 \text { al } 6 \\
2 \text { al } 6 \\
2 \text { al } 6 \\
2 \text { al } 6 \\
\end{array}$ & $\begin{array}{l}1 \text { al } 3 \\
1 \text { al } 3 \\
1 \text { y } 2 \\
1 \text { y } 2\end{array}$ & $\begin{array}{l}\text { Ausentes } \\
\text { Ausentes } \\
\text { Ausentes } \\
\text { Ausentes }\end{array}$ & $\begin{array}{l}0 \\
1 \\
1 \\
1 \\
\end{array}$ & $\begin{array}{c}1+1+1 \\
(1)+1+(1) \\
0+1+0 \\
0+1+0\end{array}$ \\
\hline
\end{tabular}


de otra considerando solo los caracteres externos, pues las diferencias se encuentran en las estructuras del petasma en los machos y en el télico en las hembras (Hendrickx y Estrada-Navarrete, 1996; Perez Farfante y Kensley, 1997; Guzmán y Wicksten, 2000).

La forma general de la antena, maxílula, maxila y primer maxilípedo son similares en los tres morfotipos analizados, además, éstas también son similares a las de G. valens? y estas a su vez a las de G. elegans. La variación en la setación de estas estructuras a través del desarrollo de los estadios mysis, no parece ser un carácter específico de comparación entre los distintos morfotipos larvales del gé- nero Gennadas descritos en la literatura (Tabla 5).

El número de segmentos de los endópodos de la maxílula y el número de lóbulos basales y coxales para la maxila y primer maxilípedo son los mismos para todas las especies (Figs. 2 a 4). Los segmentos de la antena y anténula de los tres morfotipos se observan y diferencian a partir de la mysis III, lo cual coincide con lo señalado para G. valens? (Criales y McGowan, 1993).

Las larvas analizadas presentaron setas apicales en el endópodo de la antena en todos los morfotipos desde la mysis I, diferenciándose de G. valens que no presenta esta característica sino hasta la mysis III (Tabla 5).

Tabla 5. Comparación de la fórmula setal de algunos apéndices en los diferentes estadios de mysis de Gennadas.

Table 5. Comparison of setal formula of some appendages in the different mysis stages of Gennadas.

\begin{tabular}{|l|c|c|c|c|c|c|c|c|}
\hline \multicolumn{1}{|c|}{ Apéndices } & $\begin{array}{c}\text { Gennadas } \\
\text { sp.1 } \\
\text { Presente } \\
\text { estudio }\end{array}$ & $\begin{array}{c}\text { Gennadas } \\
\text { sp.2 } \\
\text { Presente } \\
\text { estudio }\end{array}$ & $\begin{array}{c}\text { Gennadas } \\
\text { sp.3 } \\
\text { Presente } \\
\text { estudio }\end{array}$ & $\begin{array}{c}\text { G. valens? } \\
\text { Criales y } \\
\text { McGowan } \\
(1993)\end{array}$ & $\begin{array}{c}\text { Gennadas } \\
\text { sp.1 }\end{array}$ & $\begin{array}{c}\text { Gennadas } \\
\text { sp.2 }\end{array}$ & $\begin{array}{c}\text { Gennadas } \\
\text { sp.3 }\end{array}$ & $\begin{array}{c}\text { Gennadas } \\
\text { valens? }\end{array}$ \\
\hline $\begin{array}{l}\text { Antena } \\
\text { exopodito } \\
\text { endopodito }\end{array}$ & 13 & 12 & $?$ & 15 & 25 & 16 & 18 & 19 \\
\hline Maxílula & 4 & $1+3$ & $?$ & 0 & 4 & 3 & 3 & 0 \\
endopodito & $1,1,3$ & $1,1,3$ & $?$ & $1,1,2$ & 1,3 & 1,0 & 1,1 & 1,3 \\
endito basal & 7 & 9 & $?$ & 7 & 10 & 12 & 8 & 8 \\
endito coxal & 7 & 7 & $?$ & 6 & 7 & 11 & 6 & 6 \\
\hline $\begin{array}{l}\text { Maxila } \\
\text { endopodito }\end{array}$ & 5 & 8 & $?$ & 5 & 7 & 8 & 5 & 5 \\
escafognatito & 10 & 12 & $?$ & 17 & $28 / 29$ & $20 / 22$ & $12 / 13$ & $18 / 19$ \\
\hline $\begin{array}{l}\text { Maxilipedo } 1 \\
\text { endopodito }\end{array}$ & $2,2,2$ & $2,2,1,4$ & $?$ & $3,1,2,2$, & $4,2,2,3$ & $2,2,2,4$ & $4,2,1,3$ & $4,2,22$ \\
exopodito & 7 & 7 & $?$ & 10 & 9 & 22 & 9 & 11 \\
basipodito & 12 & 10 & $?$ & 11 & 18 & 24 & 15 & 14 \\
coxapodito & 7 & 5 & $?$ & 5 & 7 & 11 & 7 & 5 \\
\hline
\end{tabular}

\begin{tabular}{|l|c|c|c|c|c|c|c|c|}
\hline \multicolumn{1}{|c|}{ Mysis III } & \multicolumn{5}{c|}{ Mysis IV } \\
\hline \multicolumn{1}{|c|}{ Apéndices } & $\begin{array}{c}\text { Gennadas } \\
\text { sp.1 }\end{array}$ & $\begin{array}{c}\text { Gennadas } \\
\text { sp.2 }\end{array}$ & $\begin{array}{c}\text { Gennadas } \\
\text { sp.3 }\end{array}$ & $\begin{array}{c}\text { Gennadas } \\
\text { valens? }\end{array}$ & $\begin{array}{c}\text { Gennadas } \\
\text { sp.1 }\end{array}$ & $\begin{array}{c}\text { Gennadas } \\
\text { sp.2 }\end{array}$ & $\begin{array}{c}\text { Gennadas } \\
\text { sp.3 }\end{array}$ & $\begin{array}{c}\text { Gennadas } \\
\text { valens? }\end{array}$ \\
\hline $\begin{array}{l}\text { Antena } \\
\text { exopodito } \\
\text { endopodito }\end{array}$ & $29 / 30$ & $27 / 28$ & $28 / 29$ & $20 / 21$ & $31 / 33$ & $34 / 35$ & $33 / 34$ & $22 / 23$ \\
\hline Maxílula & 5 & 11 & 3 & 1 & 3 & 12 & 5 & 1 \\
endopodito & 3 & 1 & 2 & 3 & 2 & 1 & 2 & 2 \\
endito basal & 11 & 12 & 16 & 9 & 11 & 15 & 17 & 10 \\
endito coxal & 10 & 12 & 14 & 7 & 10 & 12 & 15 & 9 \\
\hline Maxila & & & & & & & & \\
endopodito & 7 & 8 & 4 & 6 & 8 & 8 & 3 & 6 \\
escafognatito & $30 / 31$ & $32 / 33$ & $22 / 23$ & $20 / 21$ & $32 / 33$ & $48 / 50$ & $40 / 41$ & $22 / 23$ \\
\hline $\begin{array}{l}\text { Maxilipedo } 1 \\
\text { endopodito }\end{array}$ & $2,2,1,3$ & $5,2,2,4$ & $2,0,0,4$ & $4,0,1,3$ & $3,2,1,2$ & $5,2,0,4$ & $5,2,0,4$ & $3,2,0,4$ \\
exopodito & 15 & 22 & 12 & 12 & 20 & 22 & 16 & 14 \\
basipodito & 19 & 24 & 17 & 16 & 20 & 24 & $22 / 23$ & 18 \\
coxapodito & 9 & 11 & 10 & 6 & 9 & 11 & 15 & 6 \\
\hline
\end{tabular}


Las diferencias entre los estadios mysis de cada morfotipo siguieron el mismo desarrollo descrito por Criales y MacGowan (1993), basándose principalmente en cambios en la espinación del telson, el desarrollo de la espina antrerolateral de la antena, la segmentación de los flagelos de la antena y anténula, y el desarrollo gradual de los pereiópodos y pleópodos.

Las mysis de los tres morfotipos estudiados, exhibieron un patrón secuencial en el desarrollo de cada apéndice e incremento de setación en los apéndices. Los cambios morfológicos fueron uniformes en todos los especímenes, tanto en la reducción de la espinación como en el incremento en talla de un estadio a otro. Las mediciones efectuadas en los distintos estadios de mysis fueron similares a las registradas para G. valens? en cada estadio respectivamente.

Durante el desarrollo del programa CIMAR-5 sólo se registró una especie en estado adulto del género Gennadas en las cercanías del continente y en los alrededores de isla de Pascua, la cual fue identificada como G. barbari Vereschaka. Esta especie ha sido citada con anterioridad para la región de los montes submarinos de la placa de Nazca, y Salas y Gómez por cruceros rusos (Vereschaka, 1990).

Los ejemplares de G. barbari se capturaron en las estaciones 7, 9 y 11, por lo tanto es posible que algunas larvas analizadas pertenezcan a esta especie y con mayor probabilidad las del morfotipo Gennadas sp.1, que se capturó en casi las mismas estaciones (Tabla 2). Sin embargo, para la zona norte del país hay registradas cinco especies de Gennadas (Guzmán y Wicksten, 2000). Por otra parte, para los montes submarinos de las placas de Nazca, y de Salas y Gómez otras cuatro especies del mismo género han sido citadas (Vereschaka, 1990), por lo que asignar las larvas a una especie en particular aún no es posible.

El ciclo larval de Bentheogennema burkenroadi Krygier y Wasmer de la misma familia, indica que las larvas permanecerían en el plancton por alrededor de cinco meses, con una longevidad de dos años (Krygier y Wasmer, 1975), esta podría ser la causa de haber registrado tres morfotipos distintos, que corresponderían al menos a otras dos especies que no fueron capturadas durante el muestreo. No obstante, para aguas chilenas se ha registrado un total de ocho especies pertenecientes a esta familia, agrupadas en tres géneros (Guzmán y Wicksten, 2000), pero todas se encuentran en las primeras $100 \mathrm{mn}$ de la costa.

\section{AGRADECIMIENTOS}

Los autores expresan sus sinceros agradecimientos al Comité Oceanográfico Nacional de Chile (CONA) por el desarrollo de los cruceros de investigación científica marina CIMAR 5 y 6 , que han permitido la elaboración de trabajos que contribuyen al conocimiento de la biodiversidad marina. También agradecen a los Drs. K. Konishi de National Research Institute of Aquaculture, Japón y K. Hashizume de Ocean Research Institute de la Universidad de Tokio, Japón, por sus comentarios y literatura facilitada.

\section{REFERENCIAS}

Andersen, V. y J. Sardou. 1992. The diel migrations and vertical distributions of zooplankton and micronekton in the northwestern Mediterranean Sea. 1. Euphausiids, mysids, decapods and fishes. J. Plankton Res., 14(8): 1129-1154.

Criales, M. y M.F. McGowan. 1993. Occurrence and description of mysis larvae of a mesopelagic shrimp species of the genus Gennadas (Crustacea: Decapoda: Aristeidae) from coastal waters of south Florida. Bull. Mar. Sci., 53(3): 973-984.

Díaz, G. 1998. Description of the last seven pelagic larval stages of Squilla sp. (Crustacea, Stomatopoda). Bull. Mar. Sci., 62(3): 753-762.

Foxton, P. 1970. The vertical ditribution of pelagic decapod (Crustacea: Natantia) collected on the SOND cruise 1965 II. The Penaidea and general discussion. J. Mar. Biol. Assoc., 50: 961-1000.

Gurney, R. 1924. Decapod larvae. Part IX. British Antarctic (Terra Nova) Expedition. Nat. Hist. Rep. Terra Nova Exped., 8(2): 38-200.

Gurney, R. 1942. Larvae of Decapod Crustacea. Roy. Soc., London, 306 pp.

Guzmán, G. y M. Wicksten. 2000. The subfamily Benthesicyminae Bouvier, 1908 (Decapoda, Dendrobranchiata) in northern Chile $\left(18^{\circ}\right.$ to $\left.22^{\circ} \mathrm{S}\right)$. Crustaceana, 73(8): 925-931.

Hanamura, Y. 1983. Pelagic shrimp (Penaeidea and Caridea) from Baja California and its adjacent region with description of a new species. Bull. Biogr. Soc. Japan, 38: 51-85. 
Hart, J.F. 1971. Key to planktonic larvae of families of decapod Crustacea of British Columbia. Syesis, 4: 227-234.

Heffernam, J.J. y T.L. Hopkins. 1981. Vertical distribution and feeding of the shrimp genera Gennadas and Bentheogennema (Decapoda: Penaeidea) in the eastern Gulf of Mexico. J. Crust. Biol., 1: 461-473.

Heldt, J.H. 1938. La reproduction chez les crustacés décapodes de la famille des Penaidés. Ann. Inst. Oceanogr. Monaco, 18: 1-206.

Hendrickx, M.E. y F. Estrada-Navarrete. 1986. A checklist of the species of pelagic shrimps (Penaeoidea and Caridea) from eastern Pacific, with notes on the geographic and depth distribution. CALCOFI Rep., 30: 104-121.

Hendrickx, M.E. y F. Estrada-Navarrete. 1996. Los camarones pelágicos (Crustacea: Dendrobranchiata y Caridea) del Pacífico mexicano. CONABIO, Instituto de Ciencias del Mar y Limnología, UNAM, México, 157 pp.

Kikuchi, T. y T. Nemoto. 1986. List of pelagic shrimp (Crustacea, Decapoda) from the western North Pacific. Bull. Biogr. Soc. Japan, 41: 51-59.

Kikuchi, T. y T. Nemoto. 1991. Deep-sea shrimp of genus Benthesicymus (Decapoda: Dendrobranchiata) from the western North Pacific. J. Crust. Biol., 11: 64-89.
Krygier, E.E. y R.A. Wasmer. 1975. Description and biology of a new species of pelagic penaeid shrimp, Bentheogennema burkenroadi, from the northeastern Pacific. Fish. Bull., 73(4): 737-746.

Omori, M. 1974. The biology of pelagic shrimps in the oceans. Adv. Mar. Biol., 12: 233-324.

Perez Farfante, I. y B. Kensley. 1997. Penaoid and sergestoid shrimps and prawns of the world, key and diagnoses for the families and genera. Editions du Muséum, Paris, 233 pp.

Subrahmanyam, C.B. y G. Gunter. 1970. New penaeid shrimp larvae from the Gulf of Mexico (Decapoda: Penaeidea). Crustaceana, 19: 94-98.

Vereschaka, A.L. 1990. Pelagic decapods from semount of Nazca and Sala-y-Gomez ridges. En: A.N. Mironov y J.A. Rudjacov (eds.). Plankton and benthos from the Nazca and Sala-y-Gomez submarine ridges. Academy of Sciences of the USSR, pp.129-155.

Wehrtmann, I.S. y P. Báez. 1997. Larvas y estadios tempranos de desarrollo de crustáceos decápodos de Chile: descripciones publicadas. Invest. Mar., Valparaíso, 25: 263-276.

Wicksten, M.K. y M.E. Hendrickx. 1992. Checklist of penaeoid and caridean shrimps (Decapoda: Penaeoidea, Caridea) from the eastern tropical Pacific. Proc. San Diego Soc. Natur. Hist., 9: 1-11. 